\title{
Morfologia de esporos de pteridófitas do Parque Estadual das Fontes do Ipiranga (São Paulo, Brasil)
}

\author{
Família: 21-Tectariaceae
}

Cynthia Lebrão ${ }^{1,2}$, Carolina Brandão Coelho ${ }^{1,2}$ e Luciano Mauricio Esteves ${ }^{1,3}$

Recebido: 19.04.2013; aceito: 22.11.2013

\begin{abstract}
Morphology of spores of pteridophyte from the Parque Estadual das Fontes do Ipiranga (São Paulo, Brazil). Family: 21-Tectariaceae). Spores of seven pteridophyte species of the family Tectariaceae from the Parque Estadual das Fontes do Ipiranga were studied. The family is represented in the area by three genera: Ctenitis, Lastreopsis and Megalastrum. Descriptions, illustrations, observations, as well as an identification key are presented.

Keywords: morphology, pteridophyte, spores, Tectariaceae
\end{abstract}

RESUMO - (Morfologia de esporos de pteridófitas do Parque Estadual das Fontes do Ipiranga (São Paulo, Brasil). Família: 21-Tectariaceae). Foram estudados os esporos de sete espécies de pteridófitas pertencentes à família Tectariaceae no Parque Estadual das Fontes do Ipiranga. A família está representada na área por três gêneros: Ctenitis, Lastreopsis e Megalastrum. Para todos os táxons estudados são apresentadas descrições, ilustrações, observações, bem como chave para a identificação das espécies.

Palavras-chave: esporos, morfologia, pteridófitas, Tectariaceae

\section{Introdução}

A família Tectariaceae possui 15 gêneros e cerca de 500 espécies, sendo sua distribuição cosmopolita e geralmente presente em regiões tropicais (Moran 1995). São plantas de hábito terrestre ou rupícola (Prado \& Hirai 2010).

Três gêneros e sete espécies ocorrem na área do Parque Estadual das Fontes do Ipiranga (PEFI): Ctenitis (C. Chr.) C. Chr. [C. aspidioides (C. Presl) Copel., C. distans (Brack) Ching e C. falciculata (Raddi) Ching], Lastreopsis Ching [L. amplíssima (C. Presl) Tindale] e Megalastrum Holttum [M. albidum R.C. Moran, M. connexum (Kaulf.) A.R. Sm. e M. crenulans (Fée) A.R. Sm.].

Os esporos da família Tectariaceae são elipsoidais a esferoidais e na maioria absoluta, monoletes. $\mathrm{O}$ exosporo é predominantemente psilado ou com leves ondulações, e normalmente mais delgado do que o perisporo. O perisporo apresenta grandes dobras, frequentemente equinadas; apresenta rugas, tubérculos e cristas em arranjos diversos, ou então apresenta projeções proeminentes (Tryon \& Lugardon 1990).

A análise morfológica dos esporos da família Tectariaceae complementa os estudos taxonômicos das espécies de pteridófitas presentes no PEFI e ampliam o material de referência para estudos de precipitação esporo-polínica e sedimentos na área.

\section{Material e métodos}

O planejamento do estudo da presente flora e os dados referentes ao Parque Estadual das Fontes do Ipiranga (PEFI) encontram-se descritos no trabalho de Melhem et al. (1981). A chave para identificação da família Tectariaceae do PEFI foi publicada em Prado

1. Instituto de Botânica, Caixa Postal 68041, 04045-972 São Paulo, SP, Brasil

2. Instituto de Botânica, Programa de Pós-Graduação em Biodiversidade Vegetal e Meio Ambiente, Caixa Postal 68041, 04045-972 São Paulo, SP, Brasil

3. Autor para correspondência: luciano.esteves@yahoo.com.br 
\& Hirai (2010). Segundo a classificação de Smith et al. (2006, 2008), baseada em filogenia molecular, os gêneros Ctenitis, Lastreopsis e Megalastrum encontram-se atualmente na família Dryopteridaceae, porém como a flora do PEFI foi iniciada em 2004, adotando a circunscrição de famílias utilizadas por Moran (1995) para a Flora Mesoamericana, foi mantida essa mesma circunscrição.

Os esporos coletados foram obtidos de material herborizado depositado no Herbário Científico do Estado "Maria Eneyda P. Kauffmann Fidalgo"(SP). Quando os espécimes apresentavam-se inférteis, ou com quantidade insuficiente de esporos, optou-se por coletas a partir de exsicatas provenientes de outras localidades.

Os esporos foram preparados segundo a técnica de acetólise (Erdtman 1960). As ilustrações foram feitas em microscopia óptica com esporos acetolisados e fotografados digitalmente, utilizando-se fotomicroscópio Primo Star Zeiss acoplado a uma câmera Canon A650 IS e microcomputador PC, sendo a captura das imagens feitas no programa Axion Vision para Windows e no microscópio óptico Olympus BX50 com câmera de vídeo (Olympus SC30) acoplada a um microcomputador PC, utilizando-se o programa CELLSENS STANDARD de análise de imagens. Foram medidos os diâmetros: equatorial maior (EM), equatorial menor $(\mathrm{Em})$, polar $(\mathrm{P})$ e o comprimento da lesão (L), determinando-se a média, o intervalo de confiança a $95 \%$ e o coeficiente de variabilidade da amostra. As descrições morfológicas dos esporos seguem a nomenclatura citada por Lellinger (2002).

As lâminas preparadas encontram-se depositadas na Palinoteca do Núcleo de Pesquisa em Palinologia, Instituto de Botânica/São Paulo (IBt/SP), Secretaria do Meio Ambiente.

\section{Resultados e Discussão}

\section{Ctenitis (C.Chr.) C. Chr.}

Esporos monoletes elipsoidais a esferoidais com lesão medindo $1 / 2$ a 3/4 de comprimento em relação ao diâmetro equatorial maior. Exosporo de duas camadas, psilado. Perisporo de camada única, com dobras, formando muitas vezes tubérculos largos ou estelas mais altas do que largas com ápice agudo (ornamentação equinada).

\section{Ctenitis aspidioides (C. Presl) Copel.} Figuras 1-3

Forma: monolete, plano-convexo.

Lesão: aproximadamente $1 / 3$ do diâmetro equatorial maior.
Superfície: Exosporo psilado. Perisporo apresenta grandes dobras, que formam longas cristas, com deposição de material granulado que é removido pela acetólise.

Medidas $(\mu \mathrm{m}):$ J. Prado \& D.M. Vital 1442: $\mathrm{P}=29,4 \pm 1,7 ; \mathrm{EM}=39,3 \pm 1,1 ; \mathrm{Em}=26,7 \pm 1,2 ;$ $\mathrm{L}=23,7 \pm 2,0$.

W. Hoehne 2647: $\mathrm{P}=28,9 \pm 1,8 ; \mathrm{EM}=44,6 \pm 2,3 ;$ $\mathrm{Em}=29,0 \pm 2,0 ; \mathrm{L}=24,4 \pm 1,0$.

O. Handro 2179: $\mathrm{P}=26,8 \pm 2,2 ; \mathrm{EM}=39,1 \pm 2,6$; $\mathrm{Em}=28,1 \pm 2,2 ; \mathrm{L}=20,4 \pm 2,7$.

O. Handro 2208: $\mathrm{P}=27,3 \pm 1,2 ; \mathrm{EM}=39,5 \pm 2,7$; $\mathrm{Em}=26,3 \pm 1,5 ; \mathrm{L}=19,9 \pm 2,4$.

J.A. Corrêa 15: $\mathrm{P}=30,3 \pm 1,2 ; \mathrm{EM}=43,3 \pm 0,9 ;$ $\mathrm{Em}=30,2 \pm 1,6 ; \mathrm{L}=24,3 \pm 2,7$.

J.A. Corrêa 137: $\mathrm{P}=33,8 \pm 2,7 ; \mathrm{EM}=48,2 \pm 2,8 ;$ $\mathrm{Em}=32,3 \pm 2,9 ; \mathrm{L}=24,7 \pm 1,8$.

J. Prado \& G.B. Silva 1617: $\mathrm{P}=25,5 \pm 1,6$; $\mathrm{EM}=39,2 \pm 2,0 ; \mathrm{Em}=25,6 \pm 3,6 ; \mathrm{L}=20,4 \pm 2,7$.

Observação: o material, F.C. Hoehne s.n. (SP35630), citado por Prado (2010), não foi estudado por apresentar esporos em quantidade insuficiente para análise.

Material examinado: 10-VIII-1948, W. Hoehne 2647 (SPF); 4-XI-1971, O. Handro 2179 (SPF); 2-VI-1972, O. Handro 2208 (SPF); 30-IV-1974, J.A. Corrêa 15 (SP); 2-VI-1976, J.A. Corrêa 137 (SP); 6-X-2003, J. Prado \& D.M. Vital 1442 (SP); 20-XII-2005, J. Prado \& G.B. Silva 1617 (SP).

\section{Ctenitis distans (Brack) Ching}

Figuras 4-6

Forma: monolete, plano-convexo.

Lesão: aproximadamente $1 / 3$ do diâmetro equatorial maior.

Superfície: Exosporo psilado. Perisporo apresenta grânulos e pequenas pregas, que eventualmente formam pequenas cristas.

Medidas $(\mu \mathrm{m}):$ G. Eiten \& L.T. Eiten 2080: $\mathrm{P}=22,2 \pm 0,9 ; \mathrm{EM}=35,9 \pm 1,1 ; \mathrm{Em}=26,9 \pm 1,9 ;$ $\mathrm{L}=23,8 \pm 1,6$.

O. Handro 2213: $\mathrm{P}=25,4 \pm 1,4 ; \mathrm{EM}=36,7 \pm 1,3$; $\mathrm{Em}=24,8 \pm 1,3 ; \mathrm{L}=20,0 \pm 2,0$.

Material examinado: 13-VII-1960, G. Eiten \& L.T. Eiten 2080 (SP) 7-XI-1972, O. Handro 2213 (SP).

\section{Ctenitis falciculata (Raddi) Ching}

Figuras 7-9

Forma: monolete, plano-convexo.

Lesão: aproximadamente $1 / 3$ do diâmetro equatorial maior. 

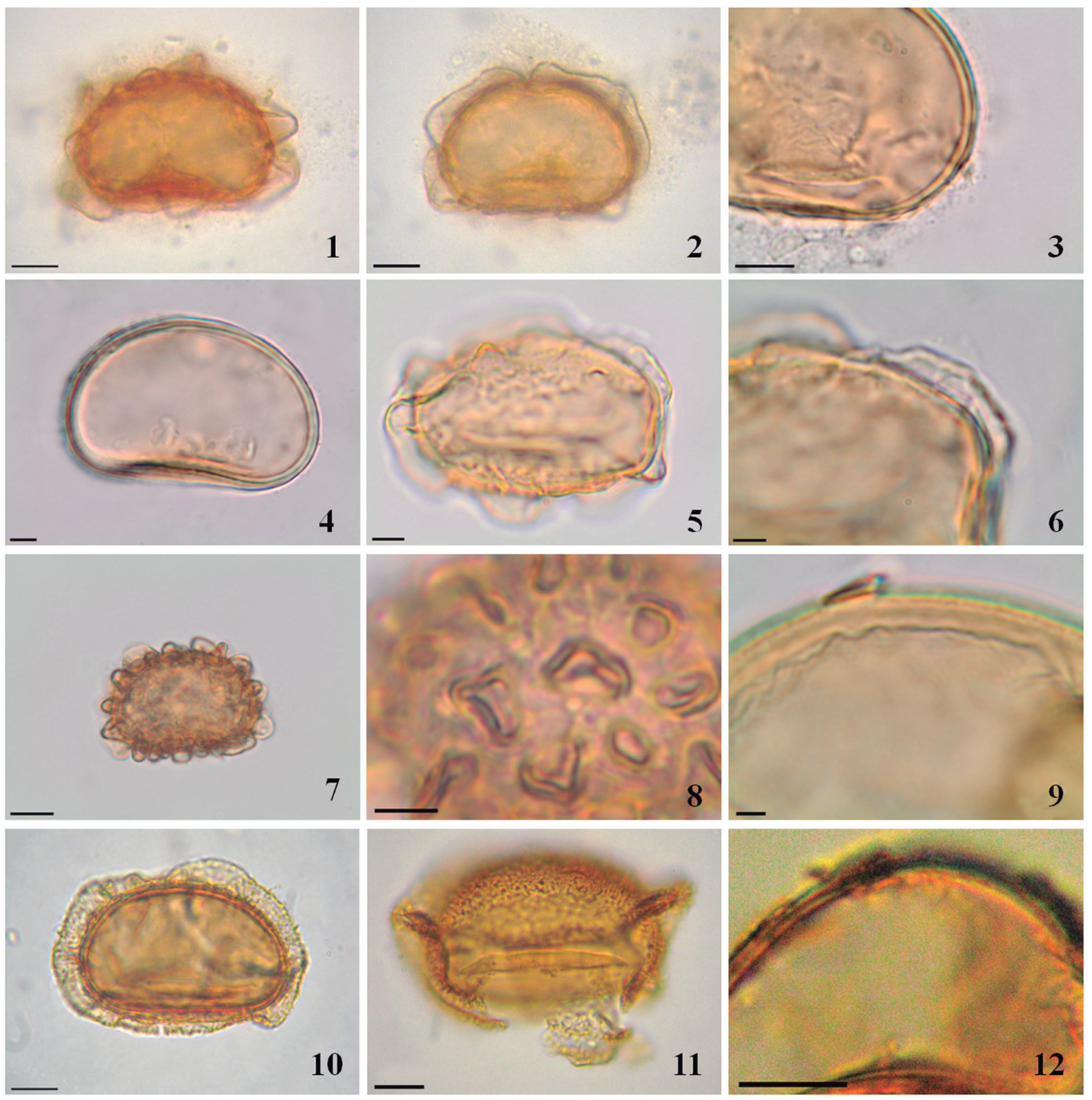

Figuras 1-12. Fotomicrografias dos esporos dos gêneros Ctenitis (C.Chr.) C.Chr. e Lastreopsis Ching (Tectariaceae). Figuras 1-3. Ctenitis aspidiodes (C.Presl) Copel. 1. Vista equatorial. 2. Vista polar distal, detalhe por transparência da lesão. 3. Corte óptico da parede. Figuras 4-6. Ctenitis distans (Brack.) Ching 4. Vista equatorial, exosporo psilado. 5. Vista polar distal, detalhe por transparência da lesão e do perisporo com pregas. 6. Corte óptico da parede, eveidenciando perisporo. Figuras 7-9. Ctenitis falciculata (Raddi) Ching. 7. Vista equatorial. 8. Detalhe do perisporo. 9. Corte óptico da parede. Figuras 10-12. Lastreopsis amplíssima (C. Presl) Tindale. 10. Vista equatorial. 11. Vista polar proximal, detalhe da lesão. 12. Corte óptico da parede. Escala nas figuras $9=2 \mu \mathrm{m} ; 6=5 \mu \mathrm{m} ; 1-5,7-8,10-12=10 \mu \mathrm{m}$.

Figures 1-12. Photomicrographs of spores of the genera Ctenitis (C.Chr.) C.Chr. and Lastreopsis Ching (Tectariaceae). Figures 1-3. Ctenitis aspidiodes (C. Presl) Copel. 1. Equatorial view. 2. Distal polar view, detail of the laesure by transparency. 3. Optical section of the wall. Figures 4-6. Ctenitis distans (Brack.) Ching. 4. Equatorial view, psilate exospore. 5. Distal polar view, detail of the laesure by transparency and perispore with folds. 6. Optical section of the wall, evidencing perispore. Figures 7-9. Ctenitis falciculata (Raddi) Ching. 7. Equatorial view. 8. Perispore detail. 9. Optical section of the wall. Figures 10-12. Lastreopsis amplissima (C. Presl) Tindale. 10. Equatorial view. 11. Proximal polar view, detail of the laesure. 12. Optical section of the wall. Scale bars - Figures $9=2 \mu \mathrm{m} ; 6=5 \mu \mathrm{m}$; $1-5,7-8,10-12=10 \mu \mathrm{m}$. 
Superfície: Exosporo psilado. Perisporo apresenta pequenas dobras, que formam alguns prolongamentos semelhantes a alas.

Medidas $(\mu \mathrm{m}): \mathrm{P}=28,3 \pm 1,0 ; \mathrm{EM}=37,4 \pm 1,2 ;$ $\mathrm{Em}=27,1 \pm 1,2 ; \mathrm{L}=22,4 \pm 1,1$.

Observação: o material M. Kuhlmann s.n. (SP154340), citado por Prado (2010), não foi estudado por apresentar esporos em quantidade insuficiente para análise.

Material examinado: 12-XII-1995, D. Graçano 35 \& A.A. Azevedo (SP).

\section{Lastreopsis Ching}

Esporos monoletes elipsoidais a esferoidais com lesão medindo $2 / 3$ a $3 / 4$ de comprimento em relação ao diâmetro equatorial maior. Exosporo de duas camadas, psilado. Perisporo formado por uma única camada, reticulado, com dobras proeminentes podendo apresentar cristas equinadas.

\section{Lastreopsis amplissima (C. Presl) Tindale} Figuras 10-12

Forma: monolete, plano-convexo.

Lesão: aproximadamente $1 / 3$ do diâmetro equatorial maior.

Superfície: Exosporo psilado. Perisporo reticulado, com dobras pronunciadas formando cristas equinuladas, com pequenas estelas cônicas pontiagudas.

Medidas $(\mu \mathrm{m}):$ J. Prado 1459: $\mathrm{P}=29,9 \pm 1,7$; $\mathrm{EM}=46,6 \pm 1,7 ; \mathrm{Em}=32,1 \pm 1,3 ; \mathrm{L}=24,3 \pm 2,0$.

F.C. Hoehne s.n. (SP30028): $\mathrm{P}=33,8 \pm 1,9$; $\mathrm{EM}=50,1 \pm 1,5 ; \mathrm{Em}=33,7 \pm 3,0 ; \mathrm{L}=23,8 \pm 3,2$.

G. Eiten et al. 2085: $\mathrm{P}=30,8 \pm 4,0$; $\mathrm{EM}=50,6 \pm 2,3 ; \mathrm{Em}=27,6 \pm 2,0 ; \mathrm{L}=19,2 \pm 1,5$.

O. Handro 2222: $\mathrm{P}=29,8 \pm 3,2 ; \mathrm{EM}=42,9 \pm 3,0$; $\mathrm{Em}=24,8 \pm 1,9 ; \mathrm{L}=26,6 \pm 2,6$.

J. Prado \& D.M. Vital 1438: $\mathrm{P}=38,9 \pm 6,9$; $\mathrm{EM}=43,2 \pm 4,3 ; \mathrm{Em}=37,3 \pm 3,3 ; \mathrm{L}=24,5 \pm 2,0$.

J. Prado \& G.B. Silva 1620: $\mathrm{P}=34,1 \pm 2,0$; $\mathrm{EM}=51,1 \pm 2,7 ; \mathrm{Em}=28,5 \pm 3,2 ; \mathrm{L}=20,3 \pm 4,0$.

J. Prado \& R.Y. Hirai 2021: $\mathrm{P}=31,8 \pm 3,0 ; \mathrm{EM}=$ $45,7 \pm 3,0 ; \mathrm{Em}=31,6 \pm 2,3 ; \mathrm{L}=23,3 \pm 3,3$.

Material examinado: 5-IX-1932, F.C. Hoehne s.n. (SP30028); 13-VII-1960, G. Eiten et al. 2085 (SP); 25IV-1973, O. Handro 2222 (SPF); 5-II-2004, J. Prado 1459 (SP); 6-X-2003, J. Prado \& D.M. Vital 1438 (SP); 20-XII-2005, J. Prado \& G.B. Silva 1620 (SP); 10-II-2009, J. Prado \& R. Y. Hirai 2021 (SP).

\section{Megalastrum Holttum}

Esporos monoletes elipsoidais a esferoidais com lesão medindo $1 / 4$ a $2 / 3$ de comprimento em relação ao diâmetro equatorial maior, geralmente de difícil visualização devido à ornamentação. Exosporo de duas camadas, psilado. Perisporo formado por uma única camada compacta, equinado, ou então com cristas ou alas largas, paralelas, com as bordas denteadas.

1. Megalastrum albidum R. C. Moran, J. Prado \& Labiak

Figuras 13-15

Forma: monolete, plano-convexo.

Lesão: aproximadamente $1 / 3$ do diâmetro equatorial maior.

Superfície: Exosporo psilado. Perisporo equinado, com estelas livres, mais altas do que largas.

Medidas $(\mu \mathrm{m}): \mathrm{P}=24,2 \pm 1,2 ; \mathrm{EM}=33,2 \pm 1,5 ;$ $\mathrm{Em}=26,0 \pm 1,1 ; \mathrm{L}=16,0 \pm 1,7$.

Observações: o material J. Prado \& G.B. Silva 1616, citado por Prado (2010), não foi estudado por apresentar esporos em quantidade insuficiente para análise.

Material examinado: 11-IX-2011, E. Valduga 90 (RB).

2. Megalastrum connexum (Kaulf.) A. R. Sm. \& R. C. Moran

Figuras 16-18

Forma: monolete, plano-convexo.

Lesão: aproximadamente $1 / 2$ do diâmetro equatorial maior.

Superfície: Exosporo psilado e de difícil visualização. Perisporo cristado-fimbriado; formando cristas proeminentes com bordas de aspecto equinado e que se anastomosam na base.

Medidas ( $\mu \mathrm{m}):$ O. Handro 2193: $\mathrm{P}=29,2 \pm 0,9$; $\mathrm{EM}=39,3 \pm 1,2 ; \mathrm{Em}=30,9 \pm 1,4 ; \mathrm{L}=18,8 \pm 1,2$.

J. Prado \& R.Y. Hirai 2005: $\mathrm{P}=27,0 \pm 1,9$; $\mathrm{EM}=39,5 \pm 2,4 ; \mathrm{Em}=27,1 \pm 1,3 ; \mathrm{L}=19,5 \pm 2,0$.

Material examinado: 31-I-1972, O. Handro 2193 (SP); 1-VIII-2008, J. Prado \& R. Y. Hirai 2005 (SP).

3. Megalastrum crenulans (Fée) A. R. Sm. \& R. C. Moran

Figuras 19-21

Forma: monolete, plano-convexo.

Lesão: aproximadamente 1/3 diâmetro equatorial maior.

Superfície: Exosporo psilado. Perisporo equinado com estelas delgadas e curtas, facilmente removido pela acetólise.

Medidas $(\mu \mathrm{m}): \mathrm{P}=28,7 \pm 4,0 ; \mathrm{EM}=38,3 \pm 1,8 ;$ $\mathrm{Em}=20,6 \pm 3,5 ; \mathrm{L}=20,6 \pm 3,5$.

Material examinado: 16-V-1973, O. Handro 2224 (SP). 

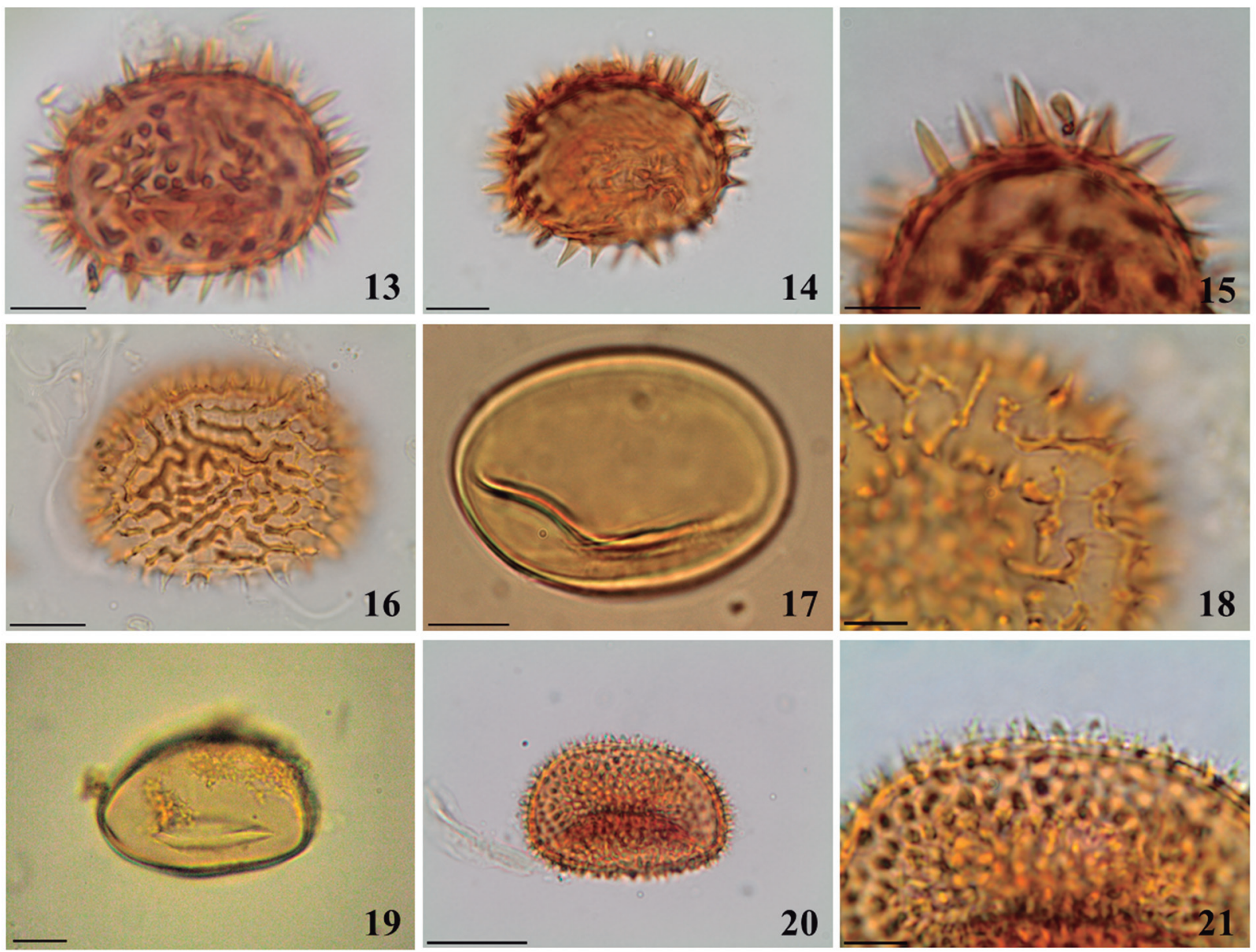

Figuras 13-21. Fotomicrografias de esporos dos gêneros Megalastrum (Tectariaceae). Figuras 13-15. Megalastrum albidum R.C. Moran, J.Prado \& Labiak. 13. Vista polar distal, perisporo equinado. 14. Vista polar distal, corte optico. 15. Corte óptico da parede. Figuras 16-18. Megalastrum connexum. 16. Vista polar distal, perisporo cristado-fimbriado. 17. Vista polar proximal, exosporo psilado. 18. Corte óptico da parede. Figuras 19-21. Megalastrum crenulans. 19. Vista polar proximal, exosporo psilado e perisporo fraturado. 20. Vista polar proximal, perisporo equinado. 21 . Corte óptico da parede. Escala nas figuras 15, 18, $21=5 \mu \mathrm{m} ; 13-14,16-17,19=10 \mu \mathrm{m} ; 20=20 \mu \mathrm{m}$.

Figures 13-21. Photomicrographs of spores of the genus Megalastrum (Tectariaceae). Figures 13-15. Megalastrum albidum R. C. Moran, J.Prado \& Labiak. 13. Distal polar view, echinate perispore. 14. Distal polar view, optical section. 15. Optical section of the wall. Figures 16-18. Megalastrum connexum. 16. Distal polar view, cristate-fimbriate perispore. 17. Proximal polar view, psilate exospore. 18. Optical section of the wall. Figures 19-21. Megalastrum crenulans. 19. Proximal polar view, psilate exospore and fractured perispore. 20. Proximal polar view, echinate perispore. 21 . Optical section of the wall. Scale bars - Figures $15,18,21=5 \mu \mathrm{m} ; 13-14,16-17,19=10 \mu \mathrm{m} ; 20=20 \mu \mathrm{m}$.

Chave para as espécies de Tectariaceae

1. Perisporo cristado-fimbriado Megalastrum connexum

1. Perisporo não cristado-fimbriado

2. Perisporo reticulado, com dobras formando cristas equinuladas Lastreopsis amplissima

2. Ausência de retículos

3. Perisporo equinado

4. Estelas livres, mas altas do que largas Megalastrum albidum

4. Estelas delgadas e curtas

3. Perisporo não equinado

5. Perisporo com pequenas dobras, formando alas Megalastrum crenulans

5. Perisporo formado por depósito de material granulado

6. Associado a dobras e longas cristas

Ctenitis falciculata

6. Associado a pregas Ctenitis aspidiodes Ctenitis distans 


\section{Agradecimentos}

Os autores agradecem ao Dr. Jefferson Prado, pela identificação do material; ao CNPq, pela bolsa PIBIC concedida à primeira Autora.

\section{Literatura citada}

Erdtman, G. 1960. The acetolysis method. A revised description. Svensk Botanisk Tidskrift 39: 561-564.

Lellinger, D.B. 2002. A modern multilingual glossary for taxonomic Pteridology. The American Fern Society, v.3.

Melhem, T.S., Giulietti, M., Forero, E., Barroso, G.M., Silvestre, M.S.F., Jung, S.L., Makino, H., Fiuza de Melo, M.M.R., Chiea, S.C., Wanderley, M.G.L., Kirizawa, M. \& Muniz, C. 1981. Planejamento para a elaboração da "Flora Fanerogâmica da Reserva do Parque Estadual das Fontes do Ipiranga (São Paulo, Brasil)". Hoehnea 9: 63-74.
Moran, R.C. 1995. Tectariaceae. In: R.C. Moran \& R. Riba (eds.). Psilotaceae a Salviniaceae. In: G. Davidse, M.S. Sousa \& S. Knapp (eds.). Flora Mesoamericana. Universidade Nacional Autónoma de México, Ciudad de México, v.1, pp. 195-470.

Prado, J. \& Hirai, R.Y. 2010. Criptógamos do Parque Estadual das Fontes do Ipiranga, São Paulo, SP. Pteridophyta: 21. Tectariaceae. Hoehnea 37: 367-376.

Smith, A.R., Pryer, K.M., Schuettpelz, E., Korall, P., Schneider, H. \& Wolf, P.G. 2006. A classification for extant ferns. Taxon 55: 705-731.

Smith, A.R., Pryer, K.M., Schuettpelz, E., Korall, P., Schneider, H. \& Wolf, P.G. 2008. Fern classification. In: T.A. Ranker \& C.H. Haufler (eds.). Biology and evolution of ferns and Lycophytes. Cambridge University Press, Cambridge, pp. 417-467.

Tryon, A.F. \& Lugardon, B. 1990. Spores of the Pteridophyta. Surface, wall structure, and diversity based on electron microscope studies. Springer- Verlag, New York. 